\title{
DISEÑO Y CONSTRUCCIÓN DE SILLA DE RUEDAS MANUAL A BASE DE GUADUA ANGUSTIFOLIA KUNTH
}

\section{DESIGN AND CONSTRUCTION OF WHEELCHAIR MANUAL BASED ON GUADUA ANGUSTIFOLIA KUNTH}

\section{MSc. Ing. Nelson Iván Villamizar Cruz *, MSc. Ing. Alonso Hernández Molano * \\ PhD. Yolanda Torres Pérez**, PhD (c). MSc. Edwin B. Rua Ramírez*, Est. Cristopher Fabián López Niño}

\author{
* Facultad de Ingeniería Mecánica, Universidad Santo Tomás Seccional Tunja, Sede Centrc \\ Cll. 19 N$^{\circ} 11-64$, Tunja, Boyacá - Colombia. \\ Teléfono PBX: (57) (8) 744 0404, Línea Nacional: 01-8000-932340 \\ E-mail: \{nelson.villamizar, alonso.hernandez, edwin.rua, \\ cristopher.lopez\}@usantoto.edu.co. \\ ** Escuela de Ingeniería Electromecánica, Grupo de Investigación en Energía y Nuevas \\ Tecnologías (GENTE), Universidad Pedagógica y Tecnológica de Colombia, Facultad \\ Sede Duitama \\ Cra 18 Cll. 22, Duitama, Boyacá - Colombia \\ Teléfono 7602181 Ext: 2818 \\ E-mail: yolanda.torres01@uptc.edu.co
}

Resumen: En Colombia, el 29,32 \% de personas tienen limitación permanente para poder moverse o caminar, censo 2005 [Gómez Beltrán, 2008], Las personas que presentan algún tipo de discapacidad permanente o temporal en la parte inferior del cuerpo, requieren de silla de ruedas para su movilidad. A través de este proyecto, nosotros logramos diseñar y construir una silla de ruedas manual, liviana y económica, utilizando como material estructural la Guadua Angustifolia Kunth. Para medir la resistencia mecánica de la Guadua, nosotros realizamos pruebas de tensión, compresión y flexión en el laboratorio de la Universidad Pedagógica y tecnológica de Colombia sede Tunja, Se obtuvo la gráfica de esfuerzo vs deformación a través de una cámara termográfica y el programa Flir tools. las uniones hechas en guadua se sometieron a pruebas de carga (tracción). Finalmente se construyó la silla de ruedas a base de Guadua resultando ser liviana, cómoda y segura.

Palabras clave: Ensayos destructivos, Guadua Angustifolia Kunth, resistencia mecánica, silla de ruedas, termografía.

\begin{abstract}
:
In Colombia, $29.32 \%$ of people have permanent limitation to be able to move or walk, 2005 census [Gómez Beltrán, 2008], People who present some type of permanent or temporary disability in the lower part of the body, require a wheelchair for their mobility. Through this project, we were able to design and build a manual wheelchair, light and economical, using as a structural material the Guadua Angustifolia Kunth. In order to measure the mechanical resistance of the Guadua, we performed tension, compression and flexion tests in the laboratory of the Pedagogical and Technological University of Colombia Tunja headquarters. We obtained the graph of stress vs deformation through a thermal camera and the program Flir Tools. The joints made in guadua were subjected to load tests (traction). Finally the wheelchair was built based on Guadua being light, comfortable and safe.
\end{abstract}


Keywords: Destructive tests, Guadua Angustifolia Kunth, mechanical resistance, wheelchair, thermography.

\section{INTRODUCCIÓN}

El 29,32 \% de personas tienen limitación permanente para poderse mover o caminar de un total 41.468 .384 personas en Colombia que fueron censadas en el año 2005 (Gómez Beltrán, 2008), las cuales el total de registros de personas con discapacidad en Boyacá es de 42.705, las más afectadas es el movimiento del cuerpo, manos, brazos, piernas, con un 21,7\% (Cobo Mejia, Sandoval Cuellar, \& Alvarado Rojas, 2012). Las personas que presentan algún tipo de discapacidad permanente o temporal en la parte inferior del cuerpo, requieren de sillas de ruedas para su movilidad, desarrollando diferentes tipos de sillas de acuerdo a su discapacidad. Los costos de las sillas de ruedas tubulares en acero se consiguen desde 270.000 (pesos) (Bernal Rincon, 2016) y con un peso que oscila entre 20 y $22 \mathrm{Kg}$, el precio de las sillas de ruedas puede aumentar de acuerdo al tipo y sus características requeridas. También se encuentran sillas de ruedas estándar ultralivianas con su estructura (chasis) en aluminio con un peso de 16 a 19 Kg. (Pearlman, Cooper, \& Hong, 2010) y sillas de ruedas deportivas superligeras en titanio con un peso de $9 \mathrm{~kg}$ (Connor, Liu, \& Cooper, 2008).

Hay una intensa búsqueda de nuevos materiales y procesos de fabricación que requieren menor energía y efectos contaminantes, lo que ha llamado la atención de los investigadores a recurrir a materiales naturales como fibras vegetales, catalogando la guadua como uno de los principales materiales que pueden sustituir el acero en ciertas aplicaciones (Ghavami, 2005), considerando la guadua como un recurso renovable por su rápido crecimiento, ya que sólo requiere 3 años para ser lo suficiente madura para la cosecha (Yang, Lee, Lee, \& Cheng, 2016).

Según las estadísticas del SENA Quindío y Valle del cauca tienen la mayor área de guaduales naturales y plantadas en Colombia de Guadua Angustifolia Kunth, Quindío con un total de 8.613 hectáreas (Montoya Mejía \& Bayona Ferreira, 2006, pág. 14), en una hectárea de guadua produce entre 60 y 70 metros cúbicos de madera, un metro cubico equivale a 10 guaduas, para comprender un metro cubico de guadua en el mercado se vende a \$100. (Teneche, 2013). Boyacá tuvo exportación de materias vegetales de las especies guadua y

Universidad de Pamplona bambú por un valor de 380 US\$ y Quindío con 4,958 US\$ de un total en Colombia de 17,227 US\$ (Montoya Mejía \& Bayona Ferreira, 2006, pág. 36). En el municipio de Moniquira ubicado a 65 $\mathrm{Km}$ de Tunja se encuentran cultivos de guadua no muy significativos, en algunas partes del municipio desechan la Guadua y en otras la comercializan a muy bajos precios para la construcción de artesanías u otras aplicaciones, desconociendo y desperdiciando las excelentes propiedades mecánicas de este material y su bajo coste. En el país de Zambia, que se encuentra en áfrica sureste, ellos están elaborando sillas de ruedas a partir de bambú debido a su bajo costo porque para ellos es muy difícil adquirir una en otro material (Gilbreath, Jacob, \& Oliver, 2010)

\section{MATERIALES Y METODOS}

Se realizó un estudio bibliográfico sobre las propiedades mecánicas de la Guadua, se encontró que en el municipio de Miraflores Boyacá realizaron pruebas y caracterizaron a la Guadua Angustifolia Kunth, elaborando probetas de compresión axial o paralelo a las fibras con un nudo, dando como resultado un esfuerzo máximo de $37 \mathrm{MPa}$, de igual manera probetas de tensión paralelo a las fibras con resultado de esfuerzo máximo de 112,5 MPa (Cely Moreno, Hernández Rojas, \& Gutiérrez Junco, 2012).

Para este proyecto se seleccionó la guadua Angustifolia Kunth ubicada en el municipio de Moniquira, departamento Boyacá. Se utilizó el culmo (Tallo encima de la tierra) para elaborar las diferentes piezas de prueba y fallarlas en ensayos de tensión en una máquina Universal. La mayoría de probetas tenían un diámetro promedio de 70 $\mathrm{mm}$, las restantes tenían un diámetro promedio de $30 \mathrm{~mm}$, estas últimas, se emplearon para la elaboración de las uniones de la estructura del chasis de la silla de ruedas.

3.1 Construcción de las uniones: Para la construcción de las uniones en guadua, se seleccionó la más conveniente de acuerdo al diseño de la silla (Ubidia, 2015). Luego, se sometieron a pruebas de carga (tracción), fijando un extremo de la unión perpendicular y el contrario aplicándole pesos para así determinar la unión más resistente con la menor cantidad de acero u otros materiales 
de refuerzo. En la Figura 1. se representa gráficamente la unión transversal que se tomó como referencia.

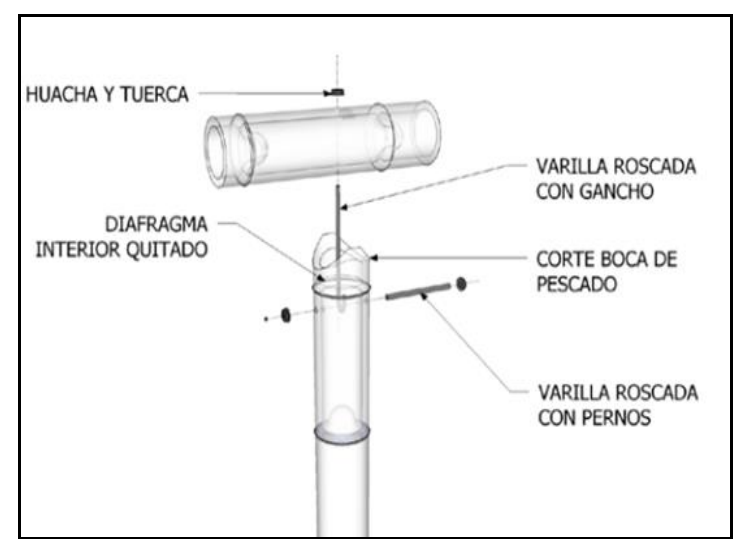

Figura 1. Representación del ensamble de la unión transversal (Ubidia, 2015)

3.2. Pruebas de ensayo: Para la preparación y ensayo de las probetas, se tomaron los tallos de guadua y se ajustaron en una fresadora universal disponible en el laboratorio 1 de la Facultad de Ingeniería Mecánica de la Universidad Santo Tomás seccional Tunja, a una velocidad de 320 rpm, siguiendo los lineamientos de la norma (NTC $5525,2007)$ en donde se indica el numeral 12.4.3 La dirección general de las fibras debe ser paralela al eje longitudinal de la porción de ensayo de la probeta, quedando así la reducción de área con unas dimensiones de 14,6 a $15,7 \mathrm{~mm}$ en la dirección tangencial. La longitud de la porción de ensayo debe estar entre 91 a $95 \mathrm{~mm}$. Ver figura 2.
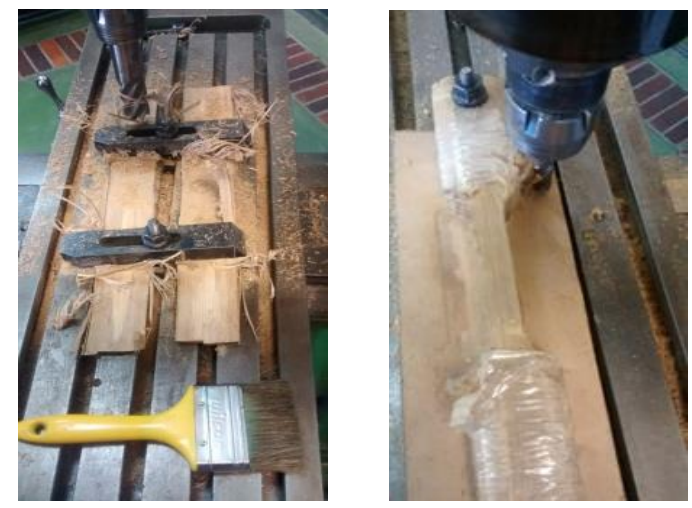

Figura 2. Preparación de las probetas

Las pruebas se realizaron en el Laboratorio de Estructuras y Resistencia de Materiales de la Universidad Pedagógica y Tecnológica de Colombia, (UPTC) a 2690 msnm, a unas condiciones del laboratorio de $17{ }^{\circ} \mathrm{C}$ de temperatura promedio y $77,3 \%$ de humedad relativa (Cely Moreno, Hernández Rojas, \& Gutiérrez Junco, 2012).

3.3 Prueba termográfica: Simultáneamente con la prueba de tensión se realizaron unas capturas a la probeta como se muestra en la Figura 3, utilizando una cámara infrarroja de marca FLIR E30 con una resolución de 160×120 pixeles, la cámara se ubicó a una distancia de 0,4 $\mathrm{m}$ desde la superficie de la probeta. La sensibilidad de la cámara es $100 \mathrm{mk}$ a $30^{\circ} \mathrm{C}$, la distancia mínima de enfoque es de $0,4 \mathrm{~m}$ en un campo de visión $25^{\circ} \times 19^{\circ}$, la resolución espacial es de 2,72 $\mathrm{mrad}$ (FLIR, 2011).

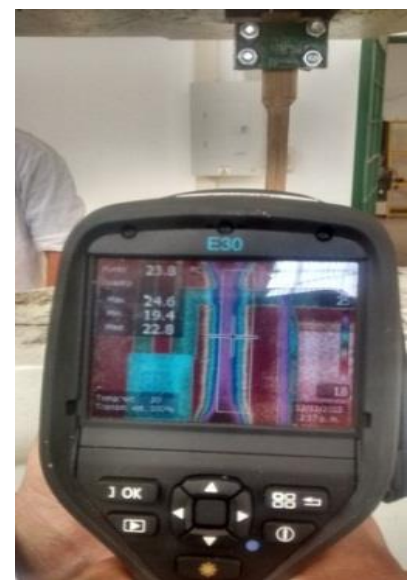

Fig. 3. Termografía aplicada a las probetas

3.4 Diseño de la estructura en Guadua: Para el diseño de la estructura o chasis se revisó la norma (NTC-ISO 9999, 1994) de "Ayudas Técnicas Para Personas Con Discapacidad" en la clasificación 12 2106 y así conocer las características básicas y necesarias que debe tener la silla de ruedas manual. Luego se propuso un diseño práctico para la estructura de la silla, teniendo en cuenta los esfuerzos a soportar cada barra o elemento de Guadua, la cantidad de uniones requeridas para unir los elementos de la estructura, el diseño estético y el tipo de ensamble de la silla. 


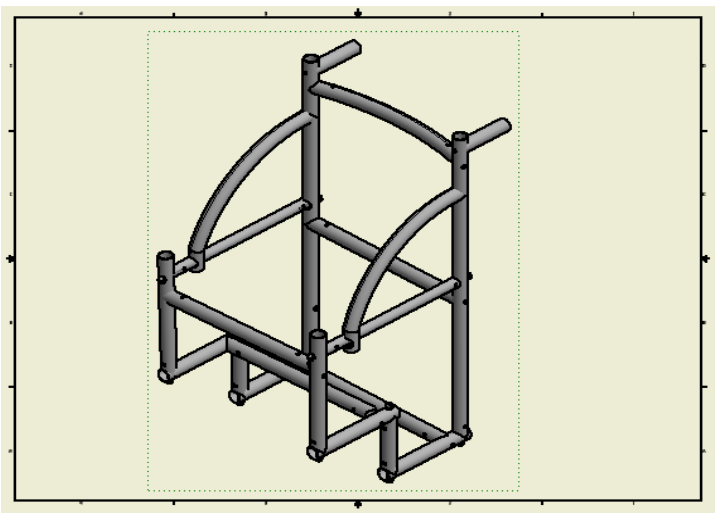

Figura 4. Diseño de estructura en guadua con programa CAD inventor 2013.

Se realizó una evaluación de los diseños propuesto y se llegó a uno final que tomó lo mejor de los modelos propuestos. Posteriormente, se elaboraron los planos de cada una de las partes de la silla de ruedas usando el programa de diseño Autodesk Inventor 2013. En la Figura 4, se puede observar el diseño de la silla y el chasis propuesto a base de guadua y algunas piezas metálicas de refuerzo estructural.

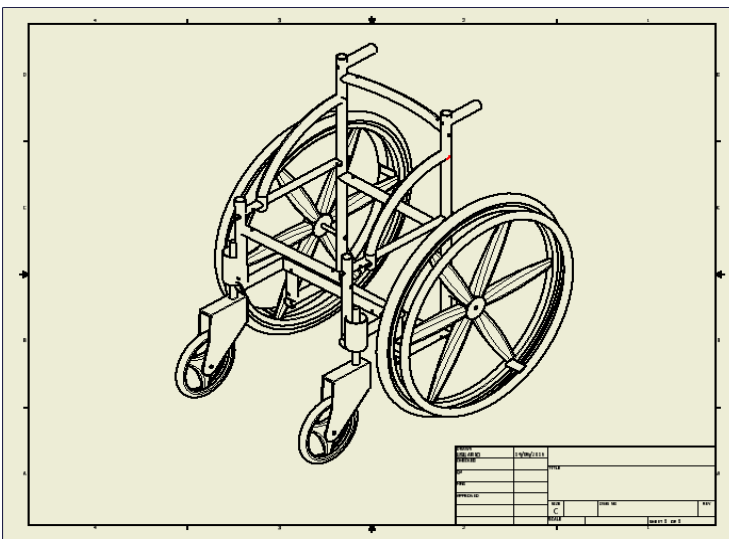

Figura 5. Ensamble de la silla de ruedas

La resistencia máxima de tensión se pudo determinar con la formula (NTC 5525, 2007).

$$
\sigma \_u l t=F \_u l t / A
$$

en donde:

$\sigma$ ult. es el esfuerzo último de tensión, en MPa (o $\mathrm{N} / \mathrm{mm} 2$ ), con aproximación de un MPa entero.

F_ult. es el valor de la carga aplicada en la cual falla la probeta, expresada en $\mathrm{N}$.

A: es el área de la sección transversal de la porción de ensayo, expresada en $\mathrm{mm} 2$.

\section{RESULTADOS}

La elaboración de la unión seleccionada consta de una platina de $1 / 4$ " roscada en la mitad, formando una abertura en las paredes a una distancia de $6 \mathrm{~cm}$ del extremo de la guadua (ver Figura 6)

En el tramo perpendicular se apoya el tornillo tensor, rellenando con una mezcla de colbón y aserrín a una longitud de $8 \mathrm{~cm}$, ver Figura 7

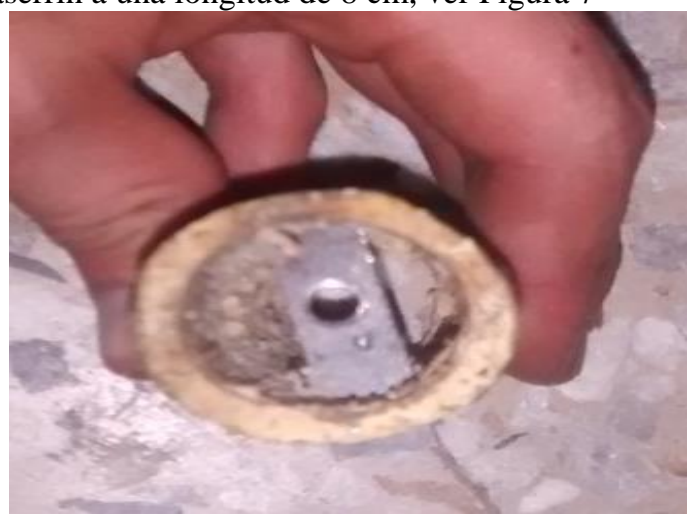

Figura 6. Ubicación de platina en la guadua

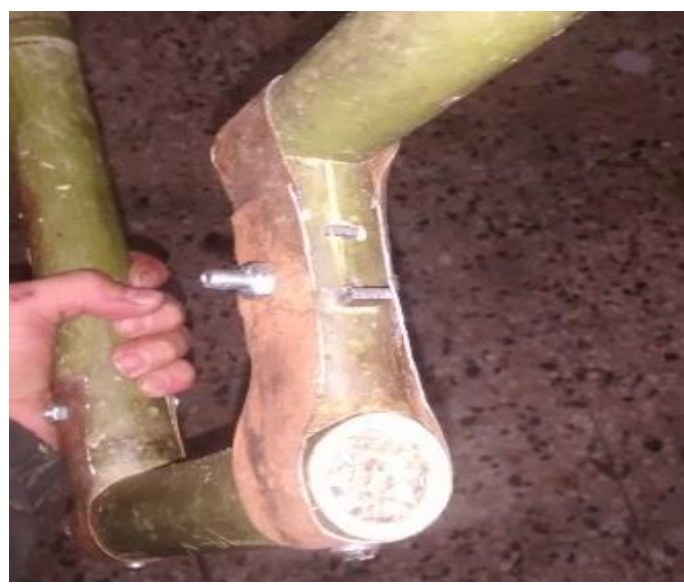

Figura 7. Ensamble de secciones de guadua.

Con las mediciones obtenidas en los ensayos de tensión y las medidas de las probetas, aplicando la ecuación 1 , se puede ver en la tabla 1, como el esfuerzo aumenta de acuerdo a la velocidad del cabezal móvil de la máquina universal. 
Tabla 1. Medidas y resultados de las pruebas de tensión.

\begin{tabular}{cccccccc}
\hline & $\begin{array}{c}\text { Espesor } \\
\text { Promedio } \\
(\mathbf{m m})\end{array}$ & $\begin{array}{c}\text { Ancho } \\
(\mathbf{m m})\end{array}$ & $\begin{array}{c}\text { Longitud } \\
(\mathbf{m m})\end{array}$ & $\begin{array}{c}\text { Velocidad } \\
(\mathbf{m m} / \mathbf{m i n})\end{array}$ & $\begin{array}{c}\text { Carga } \\
\text { Aplicada } \\
(\mathbf{N})\end{array}$ & $\begin{array}{c}\text { Área } \\
\left(\mathbf{m m}^{2}\right)\end{array}$ & $\begin{array}{c}\text { Esfuerzo } \\
\text { Último } \\
\left(\mathbf{N} / \mathbf{m m}^{2}\right)\end{array}$ \\
\hline $\mathbf{P 1}$ & $\mathbf{5 , 4}$ & 15,4 & 93 & 0,6 & 6340 & 83,16 & 76,2 \\
$\mathbf{P 2}$ & $\mathbf{3 , 1}$ & 15,7 & 92 & 1 & 8170 & 48,67 & 167,9 \\
$\mathbf{P 3}$ & $\mathbf{3 , 1}$ & 15,2 & 91,8 & 1 & 9120 & 47,12 & 193,5 \\
$\mathbf{P 4}$ & $\mathbf{3 , 1}$ & 14,7 & 92 & 8 & 9270 & 45,57 & 203,4 \\
$\mathbf{P 5}$ & $\mathbf{4 , 4}$ & 14,8 & 95 & 15 & 7700 & 65,12 & 118,2 \\
$\mathbf{P 6}$ & $\mathbf{3}$ & 14,6 & 92 & 12 & 9970 & 43,8 & 227,6 \\
\hline
\end{tabular}

Con los ensayos realizados, se construyó el diagrama esfuerzo vs deformación, tomando las tres probetas con mayor esfuerzo último, la línea azul probeta 6 , línea naranja probeta 4 y línea verde probeta 3 .

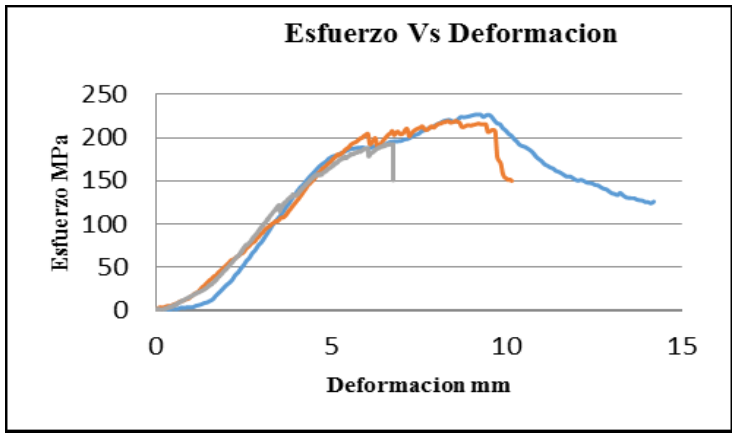

Fig. 8. Grafica esfuerzo deformación de tres probetas.

A través de la prueba termográfica se puede apreciar con colores los cambios de temperatura que soporta la probeta cuando está en su límite elástico.
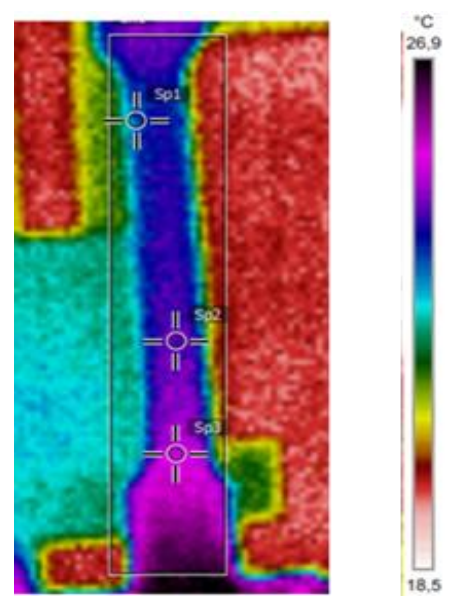

Fig. 9. Foto termográfica del límite elástico
Las fotos termográficas se analizaron en el programa Flir tools que trae la cámara, se tomaron tres puntos de referencia para medir la temperatura en los mismos, los resultados se pueden observar en la tabla 2.

Tabla 2 Resultados de temperatura Medidas

\begin{tabular}{ccc}
\hline Bx1 & Max & 26,9 \\
\hline & Min & 19,6 \\
\cline { 2 - 3 } & Promedio & 23,4 \\
\cline { 2 - 3 } Probeta 1 & & 23,4 \\
Probeta 2 & & 24,3 \\
Probeta 3 & & 25,2 \\
Parámetros & \\
\hline Emisividad & \\
Temp, refl. & & 0,95 \\
& & $50^{\circ} \mathrm{C}$
\end{tabular}

Al comienzo de la prueba la probeta tenía una temperatura promedio de $19,6^{\circ} \mathrm{C}$ y al terminar con una temperatura promedio de $26,9^{\circ} \mathrm{C}$, en las imágenes termográficas se visualiza una huella de color azul oscuro y a medida que va aumentando la carga se va desapareciendo esta huella y toma un color rosado, esto indica que está aumentando la temperatura hasta llegar a su esfuerzo último de tensión. Se construye la silla de ruedas, iniciando con la estructura propuesta la cual está conformada por 20 piezas en las cuales se encuentran 3 curvas con un total de 26 uniones utilizando el método de tornillo tensor. Para el diseño final se dejaron diferentes diámetros en la parte donde se va a ubicar el asiento de la persona, que constan de la perforación del diámetro $15 \mathrm{~mm}$ por una cara en los parales, así empotrando el travesaño perpendicular al paral con un diámetro $30 \mathrm{~mm}$ y sujetándolos con el mismo sistema de tornillo tensor.

La silla de ruedas fue construida con la menor cantidad de piezas metálicas con exención de las ruedas traseras, esta silla de ruedas cuenta con un $80 \%$ en Guadua Angustifolia Kunth, ubicada en toda su estructura. En las uniones se utilizó una cinta plástica para cubrir los tornillos que están expuestos a posibles ralladuras. 


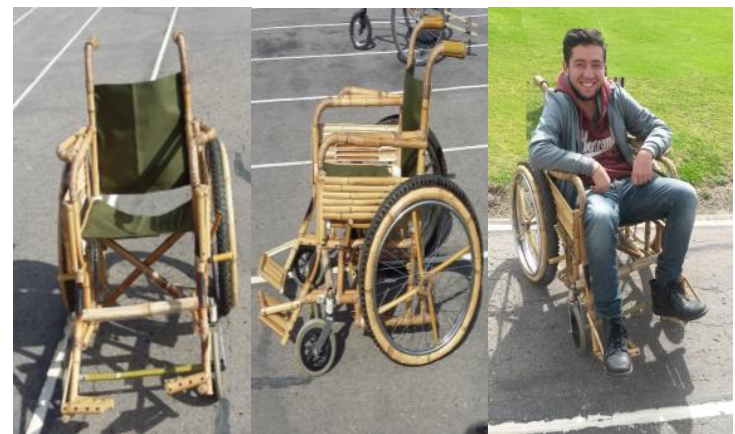

Figura 10. Silla de ruedas construida a base de guadua Angustifolia Kunth

\section{DISCUSIÓN}

En Miraflores Boyacá se realizaron pruebas a la Guadua Angustifolia Kunth, caracterizándola y obteniendo como resultado un esfuerzo máximo de 112,5 MPa (Cely Moreno, Hernández Rojas, \& Gutiérrez Junco, 2012). El acero SAE 1020 tiene una resistencia a la tensión de $441 \mathrm{MPa}$ (SAE1020, 2016), a comparación de la Guadua Angustifolia Kunth el acero es más resistente, pero la guadua tiene una menor densidad a comparación del acero. El acero es más contamínate dejando un gran rastro de desechos en toda su manufactura (Escamilla \& Habert, 2014).

\section{CONCLUSIONES}

La Guadua Angustifolia Kunth es un material natural, liviano, económico y con muy buenas propiedades mecánicas. Entre las ventajas de esta silla de ruedas manual con respecto a las de otros materiales metálicos se destaca, el precio llegando este a reducirse entre un 40 y $50 \%$ y el peso se reduce entre un 30 y $40 \%$. Cabe resaltar que esta silla de ruedas es muy práctica, liviana, cómoda y segura.

En la base del asiento es muy importante hacer un refuerzo de guadua, con el tipo de ensamble propuesto, debido a que es un punto crítico, ya que es donde se aplica la mayor parte de la carga de la persona en condición de discapacidad.

Un punto crítico en el proceso de ensamble es la sujeción de las ruedas delanteras, porque en la guadua no se pueden hacer perforaciones grandes y tampoco se pueden colocar los rodamientos de rotación de las ruedas dentro de la guadua, ya que, por la estructura física de la guadua, tiende a abrirse o partirse, por lo que es clave tener una estructura metálica sobre la guadua que permita el roscado y unión de partes de la silla.

\section{Universidad de Pamplona}

$$
\text { I. I. D. T. A. }
$$

\section{REFERENCIAS}

Bernal Rincon, A. R. (2 de marzo de 2016).

Cotizacion de silla de ruedas Standard. Tunja, Boyaca, Colombia. Obtenido de http://www.sillasderuedas.com.co/

Cely Moreno, L. A., Hernández Rojas, W. G., \& Gutiérrez Junco, O. J. (2012). Caracterizacion de la Guadua Angustifolia Kunth cultivada en Miraflores (Boyacá) de acuerdo con la NSR-10. Revista Facultad de Ingenieria, UPTC, 53-71.

Connor, S., Liu, H.-y., \& Cooper, R. A. (2008). Evaluation of titanium ultralight manual wheelchairs using ANSI/RESNA standards. Rehabilitation Research \& Development, 1251-1268.

Escamilla, E., \& Habert, G. (2014). Environmental impacts of bamboo-based construction materials representing global production diversity. Journal of Cleaner Production, 117-127.

FLIR. (2011). Flir systems Thermography product catalog. Flir Tools, 67-68.

Ghavami, K. (2005). Bamboo as reinforcement in structural concrete elements. Cement and Concrete Composites, 637-649.

Gilbreath, K., Jacob, N., \& Oliver, T. (2010). A Bamboo Wheelchair for Disabled Zambians: Phase 1-Design and Material Selection. ASME: bioengineering Division, 583-584.

Gómez Beltrán, J. C. (abril de 2008). Identificación de las personas con discapacidad. Obtenido de

DANE:

http://www.dane.gov.co/files/investigacione s/discapacidad/identificacion\%20en\%20los $\% 20$ territorios.pdf

NTC 5525. (2007). Método de ensayo para determinar las propiedades físicas y mecánicas de la Guadua Agustinofolia Kunth. 18-20. Bogotá, Colombia.

NTC-ISO 9999. (23 de noviembre de 1994). Ayudas Técnicas Para Personas Con Limitación. Bogotá, Colombia.

Pearlman, J., Cooper, R., \& Hong, E.-k. (2010). Evaluation of aluminum ultralight rigid wheelchairs versus other ultralight weelchairs using ANSI/RESNA standards. Rehabilitation Research \& Development, 441-456.

SAE-1020. (12 de 08 de 2016). Cia. General de Aceros S.A. Obtenido de http://repository.unilibre.edu.co/bitstream/h 
andle/10901/7826/VasquezTorresEdwinLib ardo2013Anexos.pdf?sequence $=2$

sillas. (12 de agosto de 2016). Sillas de ruedas. Obtenido de http://www.sillasderuedas.com.co/silla-deruedas-ultraliviana-mr1/

Smith, W. F., \& Hashemi, J. (2006). Fundamentos de la ciencia e ingeniería de materiales. México: McGRAW-HILL.

Torres, Y., Caballero C. (2014). Diseño y Fabricación del Chasis de una Silla de Ruedas Eléctrica para Niños en Condición de Discapacidad Motora de la Ciudad de Tunja. Trabajo de Grado para optar al Título de Ingeniero Mecánico. Universidad Santo Tomás Seccional Tunja.

Torres Y. (2016) "Design of Chassis and Adjustable Elements to Support Posture for Pediatric Wheelchair". Estados Unidos IFMBE Proceedings ISSN: 1680-0737, Ed: Springer Vol.60 Fasc.1 p.761 - 764 DOI: 10.1007/978-981-10-4086-3

Ubidia, J. M. (2015). MANUAL DE CONTRUCCION CON BAMBU. Lima: Red Internacional de Bambú y Ratán.

Yang, T.-h., Lee, C.-H., Lee, C.-J., \& Cheng, Y.W. (2016). Effects of different thermal modification media on physical and mechanical properties of moso bamboo. Construction and Building Materials, 251259. 